\title{
Aging and New Therapeutic Agents
}

Win MTT*

AIMST University, Malaysia

*Corresponding author: Myat Thu Thu Win, Senior Lecturer, Unit of Pharmacology, Faculty of Medicine, AIMST University, Malaysia, Tel: +60197095162; Email: mthuthusan@gmail.com

Abbreviations: AGE: advanced glycation end products; AD: Alzheimer's disease.

\section{Introduction}

Nowadays, aging disease is a burden for all countries. To prevent or treat the aging related diseases is an important part to reduce the complication of aging disease like diabetes, hypertension etc. Thus, it is very interesting issues for scientists to develop anti-aging drugs which is essential for our society. It has been described that scientists are trying to explore the drug which is beneficial for aging.

Aging is associated with an increase in the level of the advanced glycation end products (AGE) which is also related to the diabetes and cancers. The vascular injuries in diabetes are caused by both intracellular and extracellular AGE formation. There is high risk of many forms of cancers in diabetic patients. One of the commonest aging diseases Alzheimer's disease (AD) is also related to high concentration of AGE in amyloid beta $(A \beta)$ plaques in the $\mathrm{AD}$ patient's brain. So, to measure the AGE level in human disease is easy to explore the drug which will be beneficial for aging disease in our society [1].

One of the interesting Aryuvedic medicine for aging is Curcumin (Tumeric), an active component derived from Curcuma longa, is used in traditional Indian and Chinese medicines for thousands of years. Researches were done in laboratory to show the effect of turmeric properties. Some studies showed that turmeric has antioxidant, antiinflammatory and anticancer properties [2]. Moreover, turmeric possesses many medicinal uses including treatment for aging disease like Alzheimer Disease (AD). The vital constituents of turmeric are demethoxycurcumin and bisdemethoxycurcumin [3].
There was research done for curcumin effect on $A \beta$ and the results showed that curcumin prevents $A \beta$ aggregation and crosses the blood-brain barrier, reach brain cells, and protect neurons from various toxic insults of aging and $A \beta$ in humans. Oral administration of curcumin is beneficial for many diseases including diabetes, fatty-liver disease, atherosclerosis, arthritis, cancer and neurological disorders such as depression, Alzheimer's or Parkinson's disease in some studies [4]. Because of this beneficial effect, curcumin is assumed as one of the polyphenols compounds which might have effect on human aging related disease [5].

Some researchers are doing research on the wellknown antidiabetic drug, Metformin for antiaging treatment. Metformin is very useful to treat type 2 diabetes that inhibits gluconeogenesis [6]. It seems to protect against aging related diseases, cancer and inflammation [7]. One of my studies showed that metformin increased the lifespan of Caenorrhabditis elegans [8]. But, it is necessary to show the antiaging effect of metformin in human study. It is expected that new therapeutic agent for aging will be explored in near future.

\section{References}

1. Win MTT (2018) Importance of Advanced Glycation End Products (AGE) in Human Disease and Diagnosis. Advances in Bioengineering \& Biomedical Science Research journal 1(1): 1-4.

2. Unlu A, Nayir E, Kalenderoglu MD, Kirca O, Ozdogan M (2016) Curcumin (Turmeric) and cancer. J buon 21(5): 1050-1060. 


\section{Medical Journal of Clinical Trials \& Case Studies}

3. Ahmed T, Gilani AH (2014) Therapeutic potential of turmeric in Alzheimer's disease: curcumin or curcuminoids? Phytother Res 28(4): 517-525.

4. Reddy PH, Manczak M, Yin X, Grady MC, Mitchell A, et al. (2018) Protective Effects of Indian Spice Curcumin Against Amyloid- $\beta$ in Alzheimer's Disease. J Alzheimer's Dis 61(3): 843-866.

5. Ghosh SS, He H, Wang J, Gehr TW, Ghosh S (2018) Curcumin-mediated regulation of intestinal barrier function: The mechanism underlying its beneficial effects. Tissue barriers 6(1): e1425085.

6. Garg G, Singh S, Singh AK, Rizvi SI (2017) Antiaging effect of metformin on brain in naturally aged and accelerated senescence model of rat. Rejuvenation Res 20(3): 173-182.

7. Novelle MG, Ali A, Diéguez C, Bernier M, de Cabo R (2016) Metformin: a hopeful promise in aging research. Cold Spring Harbor Perspect Med 6(3): a025932.

8. Win MT, Yamamoto Y, Munesue S, Han D, Harada SI, et al. (2013) Validated liquid culture monitoring system for lifespan extension of Caenorhabditis elegans through genetic and dietary manipulations. Aging Dis 4(4): 178-185. 